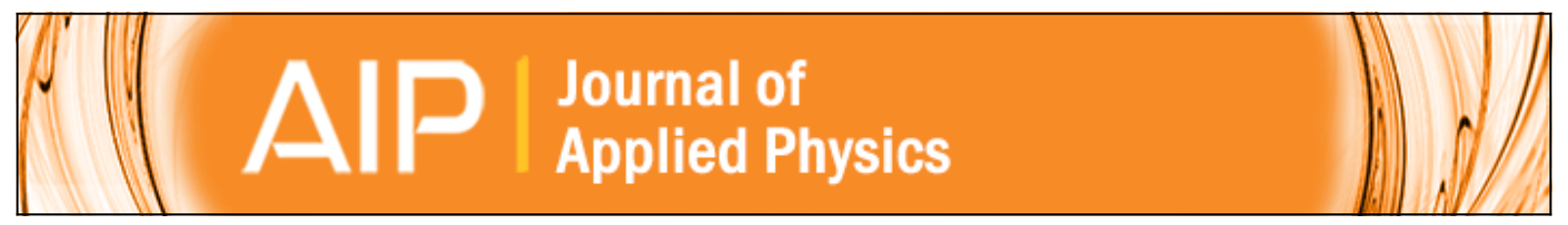

\title{
Ferromagnetism in bulk Co-Zn-O
}

A. Quesada, M. A. García, M. Andrés, A. Hernando, J. F. Fernández, A. C. Caballero, M. S. Martín-González, and $\mathrm{F}$. Briones

Citation: Journal of Applied Physics 100, 113909 (2006); doi: 10.1063/1.2399884

View online: http://dx.doi.org/10.1063/1.2399884

View Table of Contents: http://scitation.aip.org/content/aip/journal/jap/100/11?ver=pdfcov

Published by the AIP Publishing

\section{Articles you may be interested in}

Structural reconstruction and defects transition in mediating room temperature ferromagnetism in Co-doped $\mathrm{ZnO}$ film

Appl. Phys. Lett. 102, 132405 (2013); 10.1063/1.4799641

Effect of oxygen deficiency on room temperature ferromagnetism in Co doped $\mathrm{ZnO}$

Appl. Phys. Lett. 100, 202401 (2012); 10.1063/1.4717741

Strain-induced ferromagnetism enhancement in Co:ZnO films

J. Appl. Phys. 103, 093911 (2008); 10.1063/1.2919065

Enhancement of magnetic moment of Co-doped $\mathrm{ZnO}$ films by postannealing in vacuum

J. Appl. Phys. 103, 023911 (2008); 10.1063/1.2832652

Room-temperature ferromagnetism in Zn 1 - x Co x O magnetic semiconductors prepared by sputtering J. Appl. Phys. 97, 123908 (2005); 10.1063/1.1937478

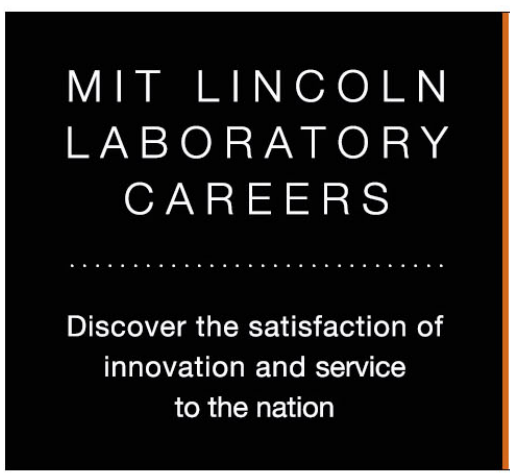

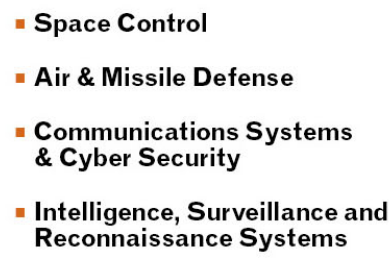

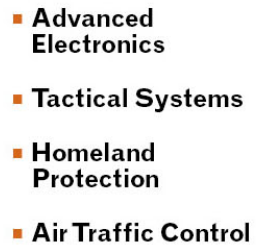

- Air Traffic Control

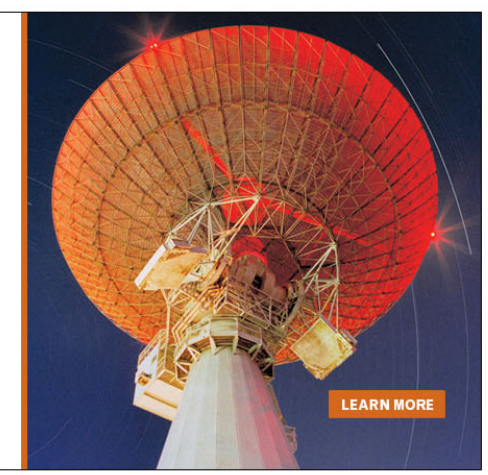




\title{
Ferromagnetism in bulk Co-Zn-O
}

\author{
A. Quesada, M. A. García, ${ }^{a}$ M. Andrés, ${ }^{\text {b) }}$ and A. Hernando \\ Instituto de Magnetismo Aplicado (RENFE-UCM-CSIC), Las Rozas, Madrid, Spain \\ and Departamento de Física de Materiales, UCM, Madrid, Spain
}

J. F. Fernández and A. C. Caballero

Instituto de Cerámica y Vidrio - CSIC, Cantoblanco, Madrid, Spain

M. S. Martín-González and F. Briones

Instituto de Microelectrónica de Madrid, CNM-CSIC, Tres Cantos, Madrid, Spain

(Received 18 July 2006; accepted 22 September 2006; published online 13 December 2006)

The origin of ferromagnetism in diluted magnetic semiconductors is still an open question, yielding a great deal of research across the world. This work focuses on the $\mathrm{Co}-\mathrm{Zn}-\mathrm{O}$ system. Room-temperature ferromagnetism is observed after a partial reaction of $\mathrm{Co}_{3} \mathrm{O}_{4}$ and $\mathrm{ZnO}$, which can be ascribed neither to carrier mediation nor segregated cobalt metallic clusters. Another mechanism is yielding room-temperature ferromagnetism. This mechanism is associated with a partial reaction of $\mathrm{ZnO}$ and $\mathrm{Co}_{3} \mathrm{O}_{4}$ grains, and always appears when the starting phases $\left(\mathrm{Co}_{3} \mathrm{O}_{4}\right.$ and $\left.\mathrm{ZnO}\right)$ are present in the sample, suggesting that interfaces are involved in the origin of the observed ferromagnetism. (C) 2006 American Institute of Physics. [DOI: 10.1063/1.2399884]

\section{INTRODUCTION}

The search for diluted magnetic semiconductors (DMS) with Curie temperature above room temperature (RT) has recently attracted much attention. According to the theoretical predictions, ${ }^{1,2}$ doping certain semiconductors with a few transition metal atoms would lead to RT ferromagnetism (FM). This FM is carrier mediated; thus, the conduction electrons would be spin-polarized, and DMS would then be excellent materials for next-generation spintronic devices. ${ }^{3-6}$ In particular, considerable work has been carried out using $\mathrm{ZnO}$ as the semiconductor matrix, as it is a wide band gap, optically transparent semiconductor, and therefore useful in many optoelectronic applications. RT FM has already been reported in $\mathrm{ZnO}$ doped with $\mathrm{Co}^{7,8}$ and with other transition metals ( $\mathrm{Ni}$ and $\mathrm{Mn}$, for example). ${ }^{9,10}$ However, the origin of ferromagnetism in these systems is still a matter of discussion. For instance, in the Mn-Zn-O system, Kundaliya et al. ${ }^{11}$ suggest that the RT FM is due to a metastable secondary phase, but we attribute it to an interface double-exchange mechanism between $\mathrm{Mn}^{3+}$ and $\mathrm{Mn}^{4+} \cdot{ }^{12}$

For the Co-Zn-O system, the situation is still more complicated as segregation of small metallic Co clusters can lead to RT FM, ${ }^{13-16}$ so the origin is not definitely established either. Some authors also claim that the FM observed is mediated by carriers in a spin-split impurity band derived from extended donor orbitals. ${ }^{17}$ A third possible origin discussed in the literature is the weak FM of $\mathrm{CoO} .{ }^{18} \mathrm{CoO}$ is well known to be antiferromagnetic, with a Néel temperature of $291 \mathrm{~K}$. FM has also been observed in bulk Co-Zn-O samples prepared by different techniques. Metallic cobalt appeared to be the origin of FM in samples prepared by the combustion method $^{19}$ because of the reduction atmosphere of the pro-

\footnotetext{
${ }^{\text {a) }}$ Electronic mail: miguelag@adif.es

b) Present address: CIEMAT Depto de Energía-Energías Renovables, Madrid, Spain.
}

cess. However, Lawes et al. $^{20}$ prepared bulk samples confirming the substitution of the magnetic ions in the semiconductor matrix and found no magnetic ordering in this system down to $2 \mathrm{~K}$. No FM has been reported in samples made by the hydrothermal technique or by standard solid-state reactions, this behavior being attributed to the absence of free carriers and the antiferromagnetic coupling of nearestneighbor cobalt ions. ${ }^{21,22}$ These two causes for the absence of FM are corroborated by theoretical works. ${ }^{15,23}$

These controversial results between research groups suggest that the FM strongly depends on the technical method used in sample preparation. Thus, in this article we will focus just on bulk samples prepared by the ceramic method, with the motivation in mind of understanding the origin of the magnetic properties in the Co-Zn-O system.

\section{EXPERIMENT}

$\mathrm{ZnO}-x\left(\mathrm{Co}_{3} \mathrm{O}_{4}\right)$ samples with $x=0.01,0.05$, and 0.25 (named ZC1, ZC5, and ZC25, respectively) were fabricated following the low-temperature procedure of Sharma et al. ${ }^{6,24}$ as follows: high-purity ( $>99.99 \%$ Aldrich) $\mathrm{ZnO}$ and $\mathrm{Co}_{3} \mathrm{O}_{4}$ raw powders were used for sample preparation. In a first step, powders were attrition milled in water medium with zirconia balls, dried, sieved, and prereacted afterward at $400{ }^{\circ} \mathrm{C}$ for $8 \mathrm{~h}$ separately in an alumina crucible. After that step, calcined powders were mixed and attrition milled again, and the dried and sieved powders pressed into disks $20 \mathrm{~mm}$ in diameter and $2 \mathrm{~mm}$ thick. The disks were then thermally treated between 500 and $1000{ }^{\circ} \mathrm{C}$ for $12 \mathrm{~h} . \mathrm{Co}_{3} \mathrm{O}_{4}$ samples, as cast, milled, and annealed following the same procedure, were also prepared for comparison purposes. The error in the determination of the samples composition is $\pm 0.5 \%$ of the total $\mathrm{Co}_{3} \mathrm{O}_{4}$ content for each sample (e.g., for the $\mathrm{ZC} 1$ sample the $\mathrm{Co}_{3} \mathrm{O}_{4}$ composition is $1 \pm 0.005 \%$ ).

The structural analysis of the samples was carried out with a Siemens D5000 x-ray diffractometer (using a mono- 


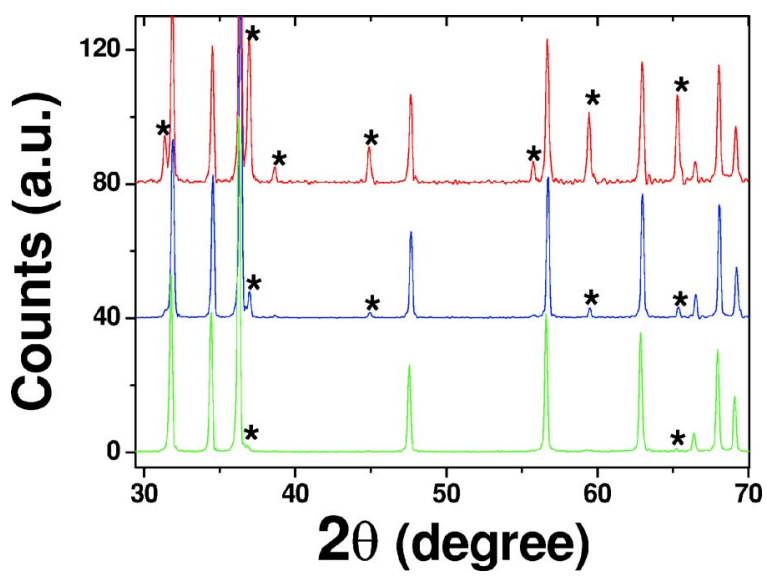

FIG. 1. (Color online) X-ray diffraction patterns for the ZC1, ZC5, and ZC25 samples after mixing by milling. $\mathrm{Co}_{3} \mathrm{O}_{4}$ diffraction maxima are indicated by asterisks; nonindexed maxima correspond to $\mathrm{ZnO}$.

chromatic $\mathrm{Cu} K \alpha$ line and operating at $40 \mathrm{kV}$ and $40 \mathrm{~mA}$ ). A vibrating sample magnetometer (VSM, JDL Instruments) was used for the magnetic characterization.

\section{RESULTS}

\section{A. As-cast starting powders}

The x-ray diffraction pattern from initial $\mathrm{ZnO}$ and $\mathrm{Co}_{3} \mathrm{O}_{4}$ powders (not shown) did not present any additional peak. Magnetic measurements of $\mathrm{ZnO}$ showed the well-known pure diamagnetic behavior, with a diamagnetic susceptibility at RT of $-1.62 \times 10^{-7}(\mathrm{emu} / \mathrm{g} \times \mathrm{Oe})$. The magnetic characterization of the $\mathrm{Co}_{3} \mathrm{O}_{4}$ powder showed the expected paramagnetic (PM) behavior at $300 \mathrm{~K}\left[\mathrm{Co}_{3} \mathrm{O}_{4}\right.$ is antiferromagnetic, with a Néel temperature of $T_{N} \sim 33 \mathrm{~K}$ (Ref. 25)] with $\chi(300 \mathrm{~K})=2.2 \times 10^{-5}(\mathrm{emu} / \mathrm{g} \times \mathrm{Oe})$, plus the presence of a small ferromagnetic signal with a saturation magnetization of $1 \times 10^{-3} \mathrm{emu} / \mathrm{g}_{\left(\mathrm{Co}_{3} \mathrm{O}_{4}\right)}$. $\mathrm{Co}_{3} \mathrm{O}_{4}$ is usually obtained by oxidation of metallic cobalt in a water vapor atmosphere. Thus, some atoms in the metallic state may remain in the sesquioxide. Taking this into account, this small ferromagnetic component could be related to the presence of Co metallic clusters in the as-cast $\mathrm{Co}_{3} \mathrm{O}_{4}$. Actually, a $0.001 \%$ of cobalt atoms in the metallic state could account for this FM. The absence of peaks corresponding to metallic cobalt in the $\mathrm{x}$-ray diffraction patterns would be justified by the fact that this percentage is well below the technique resolution. Results after the annealing processes explained below also confirmed this hypothesis.

\section{B. Milled powders}

The "so-called" ZC samples corresponding to the three compositions were studied after mixing the stoichiometric compositions by attrition milling in water medium but before any thermal treatment (not even prereacted at $400{ }^{\circ} \mathrm{C}$ ). The structural x-ray diffraction analysis from the alloyed but not yet annealed ZC samples reveals no appearance of secondary phases. Only peaks from $\mathrm{Co}_{3} \mathrm{O}_{4}$ and $\mathrm{ZnO}$ are found in the $\mathrm{X}$-ray diffraction patterns as shown in Fig. 1 .

Obviously, the increase in the $\mathrm{Co}_{3} \mathrm{O}_{4}$ concentration for the three compositions yields an increase of the peaks from

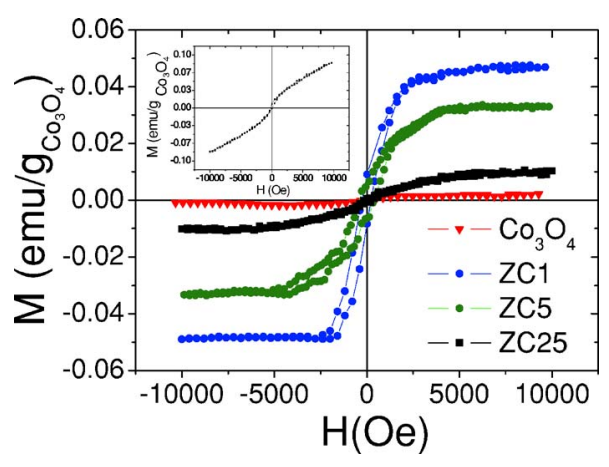

FIG. 2. (Color online) Magnetization curves at RT of the milled samples after subtracting the paramagnetic contribution. Inset shows the hysteresis loop for the ZC1 milled sample without the paramagnetic component subtraction.

$\mathrm{Co}_{3} \mathrm{O}_{4}$ in the diffraction patterns. For instance, in the $\mathrm{ZC} 1$ sample, only the two more intense peaks are observed.

Magnetization versus applied magnetic field curves were measured at $300 \mathrm{~K}(\mathrm{RT})$ for the three compositions. In the case of the ZC1 sample, a pure ferromagnetic behavior is observed in the curve. For the other two compositions and the $\mathrm{Co}_{3} \mathrm{O}_{4}$ sample, the ferromagnetic signal appears over a PM contribution. Hence, for the ZC1 sample the PM component is exactly compensated by the diamagnetic contribution of $\mathrm{ZnO}$, while for the other samples with a larger content of $\mathrm{Co}_{3} \mathrm{O}_{4}$, the PM contribution overcomes the diamagnetism from $\mathrm{ZnO}$. Figure 2 shows the magnetization curves of the three ZC samples after subtracting the PM contributions in order to display only the ferromagnetic component. Table I summarizes the values for the PM susceptibility and the ferromagnetic signal for these samples.

We observe that the ferromagnetic signal in the $\mathrm{ZC}$ samples after the mixing procedure is considerably higher than in initial $\mathrm{Co}_{3} \mathrm{O}_{4}$ (more than an order of magnitude). The highest saturation magnetization value corresponds to the $\mathrm{ZC} 1$ composition, and its value increases with the $\mathrm{ZnO}$ concentration of the samples.

In order to check if the variation of the magnetic properties during attrition milling was purely due to $\mathrm{Co}_{3} \mathrm{O}_{4}$, the initial $\mathrm{Co}_{3} \mathrm{O}_{4}$ powder alone was also milled; in this case the milling process did not modify the magnetic properties of the sample: the ferromagnetic component of this sample was exactly the same as for the as-cast $\mathrm{Co}_{3} \mathrm{O}_{4}$ $\left[0.001 \mathrm{emu} / \mathrm{g}\left(\mathrm{Co}_{3} \mathrm{O}_{4}\right)\right]$, as Fig. 2 shows.

Although the $\mathrm{x}$-ray diffraction (XRD) patterns reveal only the presence of the reactants in the sample, the increase of the FM signal after milling the samples containing $\mathrm{ZnO}$ indicates that some kind of interaction between $\mathrm{ZnO}$ and $\mathrm{Co}_{3} \mathrm{O}_{4}$ grains is taking place during the mixing process. Moreover, for milled samples, the larger FM signal corre-

TABLE I. Susceptibility of the paramagnetic component and saturation magnetization from the ferromagnetic one for the milled samples before any thermal treatment.

\begin{tabular}{ccccc}
\hline \hline Sample & $\mathrm{Co}_{3} \mathrm{O}_{4}$ & $\mathrm{ZC1}$ & $\mathrm{ZC} 5$ & $\mathrm{ZC} 25$ \\
\hline$\chi \chi_{\mathrm{PM}}(\mathrm{emu} / \mathrm{g} \times \mathrm{Oe})$ & $2.2 \times 10^{-5}$ & $1 \times 10^{-5}$ & $1.3 \times 10^{-5}$ & $2.2 \times 10^{-5}$ \\
$M_{S}(300 \mathrm{~K})(\mathrm{emu} / \mathrm{g} \times \mathrm{Oe})$ & $2 \times 10^{-3}$ & $5 \times 10^{-2}$ & $3.3 \times 10^{-2}$ & $3 \times 10^{-2}$ \\
\hline \hline
\end{tabular}




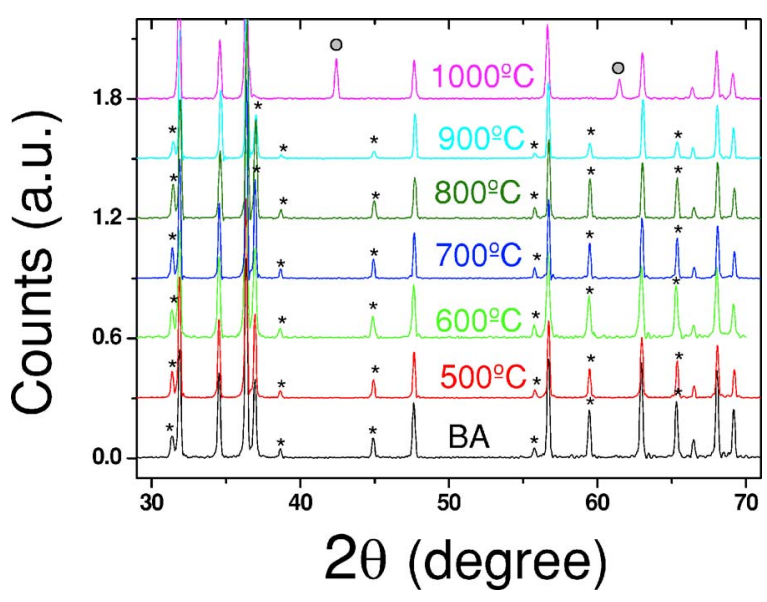

FIG. 3. (Color online) X-ray diffraction patterns from the ZC25 samples. Asterisks and circles indicate $\mathrm{Co}_{3} \mathrm{O}_{4}$ and $\mathrm{CoO}$ peaks, respectively. Nonindexed peaks correspond to $\mathrm{ZnO}$. BA stands for before annealing.

sponds to the lower PM signal (see Table I), suggesting that some initial PM phase is transforming to FM during milling. The fact that the PM susceptibility is of the same order of magnitude for the three $\mathrm{ZC}$ samples in addition to the low values of $M_{S}$ indicates that only a small fraction of the Co atoms is contributing to the ferromagnetic signal. As pure $\mathrm{Co}_{3} \mathrm{O}_{4}$ powder does not exhibit this modification of the magnetic properties after milling, such a modification involves an interaction of $\mathrm{Co}_{3} \mathrm{O}_{4}$ with $\mathrm{ZnO}$. Actually, from Table I it is clear that the modification of the magnetic properties during milling is more evident for samples with a higher $\mathrm{ZnO}$ content, confirming that the interaction with $\mathrm{ZnO}$ is responsible for the modification of the magnetic properties of $\mathrm{Co}_{3} \mathrm{O}_{4}$.

Although $0.042 \%$ of Co atoms in the metallic state in sample ZC1 would give the FM signal observed, the increase of this signal cannot be attributed to segregation of metallic Co as no mechanism yielding the formation of more metallic cobalt can occur during the mixing process. However, a presumed DMS responsible for the FM observed cannot be discarded at this point, as this partial reaction could imply the substitution of a few $\mathrm{Co}$ atoms in the $\mathrm{ZnO}$ matrix.

\section{Annealed samples}

After annealing the three $\mathrm{ZnO}$-containing samples at different temperatures in the $400-800{ }^{\circ} \mathrm{C}$ range, no changes are observed in the XRD pattern for any sample. However, as the $\mathrm{ZnCo}_{2} \mathrm{O}_{4}$ spinel is isostructural with $\mathrm{Co}_{3} \mathrm{O}_{4}$, we cannot determine from the patterns whether the spinel is forming or not.

Above $800{ }^{\circ} \mathrm{C}$, for the samples $\mathrm{ZC1}$ and $\mathrm{ZC} 5$ the maxima corresponding to $\mathrm{Co}_{3} \mathrm{O}_{4}$ disappear, but no new peaks appear. On the contrary, for sample $\mathrm{ZC} 25$, after annealing at $1000{ }^{\circ} \mathrm{C}$, the $\mathrm{Co}_{3} \mathrm{O}_{4}$ disappears from the XRD pattern and the peaks corresponding to $\mathrm{CoO}$ arise (see Fig. 3). Hence, for all the samples, after annealing at $1000{ }^{\circ} \mathrm{C}$ there is no more $\mathrm{Co}_{3} \mathrm{O}_{4}$ present in the material up to the detection limit of our equipment. No evidence of metallic $\mathrm{Co}$ or any additional phase different than $\mathrm{ZnO}, \mathrm{Co}_{3} \mathrm{O}_{4}$, and $\mathrm{CoO}$ was found for any sample up to the XRD resolution.

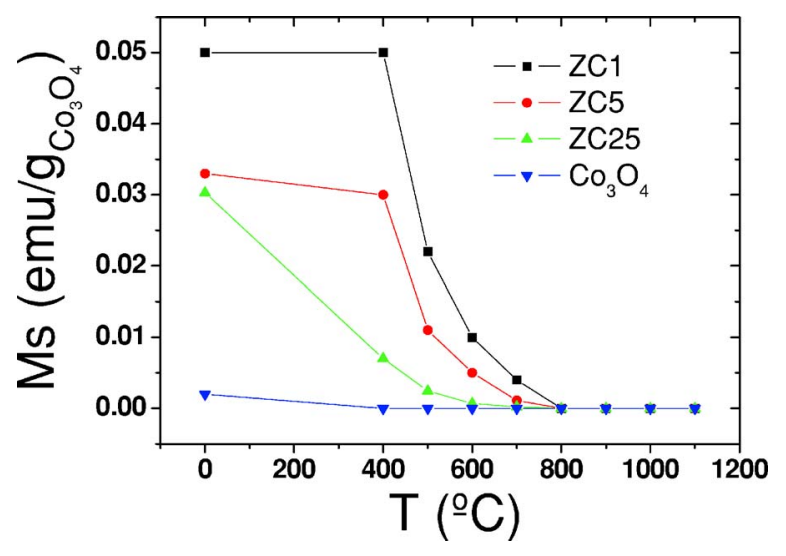

FIG. 4. (Color online) Saturation magnetization as a function of the annealing temperature.

The only reaction between $\mathrm{Co}_{3} \mathrm{O}_{4}$ and $\mathrm{ZnO}$ consistent with the XRD patterns is the formation of a solid solution $\mathrm{Zn}_{1-x} \mathrm{Co}_{x} \mathrm{O}$ with a wurtzite-type structure, with the same lattice parameter as $\mathrm{ZnO}$ that would explain the fact that only peaks from the wurtzite structure of $\mathrm{ZnO}$ are observed in the diffraction patterns above the $800{ }^{\circ} \mathrm{C}$ treatment. In the ZC25 sample, the solubility limit of $\mathrm{Co}$ in $\mathrm{ZnO}$ is reached and an excess of $\mathrm{Co}_{3} \mathrm{O}_{4}$ remains in the sample after this reaction, which reduces to $\mathrm{CoO}$ (rock-salt crystal structure) at $1000{ }^{\circ} \mathrm{C}$. This reduction is not observed for the other two compositions as evidence that all the $\mathrm{Co}_{3} \mathrm{O}_{4}$ present forms with $\mathrm{ZnO}$ the solid solution with a wurtzite-type structure mentioned earlier. The very small difference between the ionic radius of $\mathrm{Co}$ and $\mathrm{Zn}$ (75 and $74 \mathrm{pm}$, respectively) prevents us from seeing shifts in the maxima corresponding to $\mathrm{ZnO}$ wurtzite in the XRD diffraction patterns.

Annealing yields the decrease of FM for all the samples (see Fig. 4). For $\mathrm{Co}_{3} \mathrm{O}_{4}$, the FM vanishes after annealing at $400{ }^{\circ} \mathrm{C}$ (see Fig. 4), while it is still present for $\mathrm{Zn}$-containing samples after annealing up to $800{ }^{\circ} \mathrm{C}$. For $\mathrm{Co}_{3} \mathrm{O}_{4}$ it is clear that annealing in air promotes the oxidation of metallic $\mathrm{Co}$ clusters remaining in the sample, confirming the hypothesis that the ferromagnetic signal in this sample arises from those segregated clusters. The different behavior observed for the ZnO-containing samples suggests to us that the FM signal observed in these samples is not related to any metallic Co clusters, which should disappear after annealing at about $400{ }^{\circ} \mathrm{C}$. The finite mobility of oxygen in these samples that would lead to an incomplete suppression of the magnetic moment of Co does not seem a reasonable cause for the FM observed. On one side, at $400{ }^{\circ} \mathrm{C}$ the reactants have barely interacted and the $\mathrm{Co}_{3} \mathrm{O}_{4}$ environment is generally identical to that of as-cast $\mathrm{Co}_{3} \mathrm{O}_{4}$. On the other, the $\mathrm{FM}$ vanishes at $800{ }^{\circ} \mathrm{C}$ for all the samples with $\mathrm{ZnO}$, which is not consistent with the fact that the $\mathrm{ZnO}$ concentration in the sample is a key factor in the limitation of the oxygen mobility and different concentrations should lead to different vanishing temperatures of FM. Moreover, as no mechanism yields the appearance of more metallic Co in the samples during the mixing step, and after this milling the FM increases an order of magnitude its saturation magnetization value, we can thus reject the hypothesis of the segregated metallic cobalt clusters as the origin of FM in these samples. 
It is worthy to note that for $\mathrm{ZnO}$ containing samples the FM signal is present up to $800{ }^{\circ} \mathrm{C}$, the same temperature at which $\mathrm{Co}_{3} \mathrm{O}_{4}$ is still present in the sample according to the $\mathrm{x}$-ray diffraction patterns. Thus, we can conclude that the wurtzite-type solid solution formed in this reaction is not responsible for the FM. Annealing at higher temperatures would yield a greater dispersion of the $\mathrm{Co}$ ions in the $\mathrm{ZnO}$ matrix if we suppose we are dealing with a DMS. ${ }^{16}$ The decrease of FM with the subsequent anneals suggests that the origin of these magnetic properties is not associated with carrier-mediated mechanisms in a DMS.

Another possible origin invoked for the magnetic properties of this system is the weak FM of $\mathrm{CoO} .{ }^{18}$ We have also magnetically characterized $\mathrm{CoO}$, and the sample presented a pure PM behavior at RT. Moreover, the fact that the appearance of $\mathrm{CoO}$ in the $\mathrm{ZC} 25$ sample after annealing at $1000{ }^{\circ} \mathrm{C}$ corresponds to a nonferromagnetic sample allows us to put aside the $\mathrm{CoO}$ hypothesis as a possible origin of the FM observed here.

The spinel $\mathrm{ZnCo}_{2} \mathrm{O}_{4}$ can never be invoked as the origin of the observed FM: as the treatment temperature increases, the amount of spinel present in the sample should also rise (as it promotes the $\mathrm{Zn}$ further diffusion), but the FM decreases, indicating that the spinel is not the origin of the ferromagnetic component of the samples either. Thus, the FM is associated with a partial reaction in which several phases (nonferromagnetic) are present in the sample. In this situation, the interfaces could play an important role in the observed ferromagnetic behavior (as found in the case of $\mathrm{Mn}-\mathrm{Zn}-\mathrm{O}){ }^{12}$ If the FM signal arises from these interfaces, only a few atoms should contribute to the FM signal, explaining the low intensity of the FM signal. Moreover, after total reaction yielding a homogeneous composition, interfaces do not exist anymore, in agreement with the absence of FM in totally reacted samples.

We also notice that the largest saturation magnetization value corresponds to the $\mathrm{ZC} 1$ composition, indicating that the presence of $\mathrm{ZnO}$ enhances the ferromagnetic signal after alloying. This suggests that the partial reaction taking place between $\mathrm{Co}_{3} \mathrm{O}_{4}$ and $\mathrm{ZnO}$ during the mixing process, and associated with the FM observed, seems to be enhanced with $\mathrm{ZnO}$ concentration. This fact recalls the $\mathrm{Mn}-\mathrm{Zn}-\mathrm{O}$ system case, in which $\mathrm{Zn}$ diffuses into $\mathrm{MnO}_{2}$ grains and where the increase in the $\mathrm{Zn}$ concentration favors this diffusion as the chemical gradient rises, the FM appearing at the interface of the diffusion front. A limited reaction of $\mathrm{MnO}_{2}$ with $\mathrm{ZnO}$ also yields $\mathrm{FM}^{26}$ as happens in the samples studied in this work. In the case of $\mathrm{Mn}-\mathrm{Zn}-\mathrm{O}$ the double-exchange mechanism was invoked to account for the RT FM. However, double exchange is not well established for Co oxides, so this assignation is not straightforward in this case. Therefore, some so far unknown mechanism must be responsible for this FM, yet to be elucidated, and appearing after $\mathrm{Co}_{3} \mathrm{O}_{4}$ and $\mathrm{ZnO}$ partially react during the mixing step.

\section{CONCLUSIONS}

In summary, we have shown that there is a mechanism promoting RT FM in $\mathrm{Co}_{3} \mathrm{O}_{4}-\mathrm{ZnO}$ bulk samples different than carrier mediation or segregated cobalt metallic clusters. After the mixing step by milling in water medium of the starting raw powders a partial reaction takes place, giving rise to a FM component. Annealing yields the decrease of FM that is only present when several phases are present in the material, pointing out interfaces as the origin of the observed FM. Further work needs to be done in order to achieve thorough understanding of this mechanism.

\section{ACKNOWLEDGMENT}

This work has been supported by the University Complutense through the project PR1/05/13325 and CICYT project MAT2004-04843-C02-01.

${ }^{1}$ H. Ohno, Science 281, 951 (1998).

${ }^{2}$ T. Dietl, H. Ohno, F. Matsukura, J. Cibert, and D. Ferrand, Science 287, 1019 (2000)

${ }^{3}$ G. Schmidt and L. W. Molenkamp, J. Appl. Phys. 89, 7443 (2001).

${ }^{4}$ S. J. Pearton, W. H. Heo, M. Ivill, D. P. Norton, and T. Steiner, Semicond. Sci. Technol. 19, R59 (2004).

${ }^{5}$ J. M. D. Coey and S. Sanvito, J. Phys. D 37, 988 (2004).

${ }^{6}$ T. Fukumura, H. Toyosaki, and Y. Yamada, Semicond. Sci. Technol. 20, S103 (2005)

${ }^{7}$ H. J. Lee, S. Y. Jeong, C. R. Cho, and C. H. Park, Appl. Phys. Lett. 81, 4020 (2002)

${ }^{8}$ W. Prellier, A. Fouchet, B. Mercey, C. Simon, and B. Raveau, Appl. Phys. Lett. 82, 3490 (2003)

${ }^{9}$ P. V. Radovanovic and D. R. Gamelin, Phys. Rev. Lett. 91, 157202 (2003).

${ }^{10}$ P. Sharma, A. Gupta, K. V. Rao, F. J. Owens, R. Sharma, R. Ahuja, J. M. Osorio Guillen, B. Johansson, and G. A. Gehring, Nat. Mater. 2, 673 (2003).

${ }^{11}$ D.C. Kundaliya, S. B. Ogale, S. E. Lofland, S. Dhar, C. J. Metting, S. R. Shinde, Z. Ma, B. Varughese, K. V. Ramanujachari, L. Salamanca-Riba, and T. Venkatesan, Nat. Mater. 3, 709 (2004).

${ }^{12}$ M. A. García, M. L. Ruiz-González, A. Quesada, J. L. Costa-Krämer, J. F. Fernández, S. J. Khatib, A. Wennberg, A. C. Caballero, M. S. MartínGonzález, M. Villegas, F. Briones, J. M. González-Calbet, and A. Hernando, Phys. Rev. Lett. 94, 217206 (2005).

${ }^{13}$ S. R. Shinde, S. B. Ogale, J. S. Higgins, H. Zheng, A. J. Millis, V. N. Kulkarni, R. Ramesh, R. L. Greene, and T. Venkatesan, Phys. Rev. Lett. 92, 166601 (2004).

${ }^{14}$ J. H. Park, G. Kim, H. M. Jang, S. Ryu, and Y. M. Kim, Appl. Phys. Lett. 84, 1338 (2004)

${ }^{15}$ E. C. Lee and K. J. Chang, Phys. Rev. B 69, 085205 (2004).

${ }^{16}$ J. Hyun Kim, H. Kim, D. Kim, Y. Ihm, and W. K. Choo, J. Eur. Ceram. Soc. 24, 1847 (2004).

${ }^{17}$ C. B. Fitzgerald, M. Venkatesan, J. G. Lunney, L. S. Dorneles, and J. M. D. Coey, Appl. Surf. Sci. 247, 493 (2005).

${ }^{18}$ K. Ueda, H. Tabata, and T. Hawai, Appl. Phys. Lett. 79, 988 (2001).

${ }^{19}$ S. Deka and P. A. Joy, Solid State Commun. 134, 665 (2005).

${ }^{20}$ G. Lawes, A. S. Risbud, A. P. Ramírez, and R. Seshadri, Phys. Rev. B 71, 045201 (2005)

${ }^{21}$ M. Bouloudenine, N. Viart, S. Colis, and A. Dinia, Chem. Phys. Lett. 73, 397 (2004).

${ }^{22}$ S.-J. Han, B. Y. Lee, J.-S. Ku, Y. B. Kim, and Y. H. Jeong, J. Magn. Magn. Mater. 272-276, 2008 (2004).

${ }^{23}$ A. S. Risbud, N. A. Spaldin, Z. Q. Chen, S. Stemmer, and R. Seshadri, Phys. Rev. B 68, 205202 (2003).

${ }^{24}$ J. L. Costa-Krämer, F. Briones, J. F. Fernandez, A. C. Caballero, M. Villegas, M. Diaz, M. A. García, and A. Hernando, Nanotechnology 16, 214 (2005).

${ }^{25}$ Y. Ichiyanagi and S. Yamada, Polyhedron 24, 2813 (2005).

${ }^{26}$ H. J. Blythe, R. M. Ibrahim, G. A. Gehring, J. R. Neal, and A. M. Fox, J. Magn. Magn. Mater. 283, 117 (2004) 